\title{
GENERALIZED CONTRACTIONS ON DUALISTIC PARTIAL METRIC SPACES
}

\author{
MUHAMMAD NAZAM ${ }^{1, *}$, MUHAMMAD ARSHAD $^{2}$, AFTAB HUSSAIN $^{3}$ \\ ${ }^{1}$ Department of Mathematics and Statistics, International Islamic University, Islamabad 44000, Pakistan \\ ${ }^{2}$ Department of Mathematics and Statistics, International Islamic University, Islamabad 44000, Pakistan \\ ${ }^{3}$ Lahore Leads University, Department of Mathematical Sciences, Lahore - 54000, Lahore, Pakistan
}

\begin{abstract}
We introduce the notion of generalized contraction on dualistic partial metric spaces. A fixed point theorem for mappings satisfying above mentioned contraction is obtained. Some consequences of our result are obtained. We construct an example to demonstrate the effectiveness of our result among the corresponding results in partial metric spaces. Our results provide substantial generalizations and improvements of several well known results existing in the comparable literature. We discuss an application of our fixed point results to show the existence of solution of functional equations.
\end{abstract}

Keywords: Fixed point, dualistic partial metric, generalized contraction .

2010 AMS Subject Classification: 47H09; 47H10; 54H25.

\section{INTRODUCTION}

Matthews [6] introduced the concept of partial metric space (in which self distance may not be zero) as a suitable mathematical tool for program verification and proved an analogue of $\mathrm{Ba}-$ nach fixed point theorem in complete partial metric spaces. O'Neill [8] introduced the concept of dualistic partial metric, which is more general than partial metric (which allows negative values also) and established a robust relationship between dualistic partial metric and quasi metric. Oltra and Valero [7] presented a Banach fixed point theorem on complete dualistic partial metric spaces. Later on, Valero [9] generalized the main theorem of [7] using nonlinear contractive

\footnotetext{
${ }^{*}$ Corresponding author

E-mail addresses: nazim254.butt@yahoo.com (M. Nazam), marshadzia@iiu.edu.pk(M. Arshad), aftabshh@gmail.com (A. Hussain)
} 
condition instead of Banach contractive condition.

Following [2], E.Karapinar et al. [5] proved following result.

Theorem 1.1. [5] Let $(X, d)$ be a complete partial metric space and $T: X \rightarrow X$ be a mapping satisfying

$$
\varphi(p(T(x), T(y))) \leq \varphi(M(x, y))-\psi(M(x, y)) \text { for all } x, y \in X
$$

where $M(x, y)$ is given by $M(x, y)=\max \left\{p(x, y), \frac{p(y, T(y))(1+p(x, T(x))}{1+p(x, y)}\right\}$ and $\varphi:[0, \infty) \rightarrow$ $[0, \infty)$ is a continuous and monotone non-decreasing function with $\varphi(t)=0$ if and only if $t=0$ and $\psi:[0, \infty) \rightarrow[0, \infty)$ is a lower semi-continuous function with $\psi(t)=0$ if and only if $t=0$. Then $T$ has a unique fixed point.

Motivated by above cited results, in this paper, we prove a fixed point theorem on dualistic partial metric spaces and some corresponding results for a mapping satisfying generalized contraction. We present examples to illustrate our results.

\section{PRELIMINARIES}

Throughout this paper, we denote $(0, \infty)$ by $\mathbb{R}^{+},[0, \infty)$ by $\mathbb{R}_{0}^{+},(-\infty,+\infty)$ by $\mathbb{R}$ and set of natural numbers by $\mathbb{N}$. Let us recall some mathematical basics and results to make this paper self-sufficient.

Let $X$ be a nonempty set and let $T: X \rightarrow X$ be a self mapping. A point $x^{*} \in X$ is called a fixed point of $T$ if $x^{*}=T\left(x^{*}\right)$.

According to O'Neill, a dualistic partial metric can be defined as follows:

Definition 2.1. [8] Let $X$ be a nonempty set. If a function $D: X \times X \rightarrow \mathbb{R}$ satisfies, for all $x, y, z \in X$, the following properties:

$\left(D_{1}\right) x=y \Leftrightarrow D(x, x)=D(y, y)=D(x, y)$.

$\left(D_{2}\right) D(x, x) \leq D(x, y)$.

(D) $D(x, y)=D(y, x)$.

$\left(D_{4}\right) D(x, z)+D(y, y) \leq D(x, y)+D(y, z)$. 
Then $D$ is called dualistic partial metric and the pair $(X, D)$ is known as dualistic partial metric space.

Remark 2.2. It is obvious that every partial metric is dualistic partial metric but converse is not true. To support this comment, define $D_{\vee}: \mathbb{R} \times \mathbb{R} \longrightarrow \mathbb{R}$ by

$$
D_{\vee}(x, y)=x \vee y=\sup \{x, y\} \forall x, y \in \mathbb{R} .
$$

It is clear that $D_{\vee}$ is a dualistic partial metric. Note that $D_{\vee}$ is not a partial metric, because $D_{\vee}(-1,-2)=-1 \notin \mathbb{R}_{0}^{+}$. Nevertheless, the restriction of $D_{\vee}$ to $\mathbb{R}_{0}^{+},\left.D \vee\right|_{\mathbb{R}_{0}^{+}}$, is a partial metric.

Example 2.3. If $(X, d)$ is a metric space and $c \in \mathbb{R}$ is arbitrary constant, then

$$
D(x, y)=d(x, y)+c .
$$

defines a dualistic partial metric on $X$.

Following [8], each dualistic partial metric $D$ on $X$ generates a $T_{0}$ topology $\tau(D)$ on $X$. The elements of the topology $\tau(D)$ are open balls of the form $\left\{B_{D}(x, \varepsilon): x \in X, \varepsilon>0\right\}$ and $B_{D}(x, \varepsilon)=\{y \in X: D(x, y)<\varepsilon+D(x, x)\}$.

A sequence $\left\{x_{n}\right\}_{n \in \mathbb{N}}$ in $(X, D)$ converges to a point $x \in X$ if and only if $D(x, x)=\lim _{n \rightarrow \infty} D\left(x, x_{n}\right)$. If $(X, D)$ is a dualistic partial metric space, then $d_{D}: X \times X \rightarrow \mathbb{R}_{0}^{+}$defined by

$$
d_{D}(x, y)=D(x, y)-D(x, x)
$$

is called a quasi metric on $X$ such that $\tau(D)=\tau\left(d_{D}\right)$ for all $x, y \in X$. Moreover, if $d_{D}$ is quasi metric on $X$, then $d_{D}^{S}(x, y)=\max \left\{d_{D}(x, y), d_{D}(y, x)\right\}$ defines a metric on $X$.

Definition 2.4. [8] Let $(X, D)$ be a dualistic partial metric space, then

(1) A sequence $\left\{x_{n}\right\}_{n \in \mathbb{N}}$ in $(X, D)$ is called a Cauchy sequence if $\lim _{n, m \rightarrow \infty} D\left(x_{n}, x_{m}\right)$ exists and is finite.

(2) A dualistic partial metric space $(X, D)$ is said to be complete if every Cauchy sequence $\left\{x_{n}\right\}_{n \in \mathbb{N}}$ in $X$ converges, with respect to $\tau(D)$, to a point $x \in X$ such that $D(x, x)=$ $\lim _{n, m \rightarrow \infty} D\left(x_{n}, x_{m}\right)$. 
Following lemma will be helpful in the sequel.

Lemma 2.5. $[8,9]$

(1) A dualistic partial metric $(X, D)$ is complete if and only if the metric space $\left(X, d_{D}^{S}\right)$ is complete.

(2) A sequence $\left\{x_{n}\right\}_{n \in \mathbb{N}}$ in $X$ converges to a point $x \in X$, with respect to $\tau\left(d_{D}^{s}\right)$ if and only if $\lim _{n \rightarrow \infty} D\left(x, x_{n}\right)=D(x, x)=\lim _{n, m \rightarrow \infty} D\left(x_{n}, x_{m}\right)$.

(3) If $\lim _{n \rightarrow \infty} x_{n}=v$ such that $D(v, v)=0$ then $\lim _{n \rightarrow \infty} D\left(x_{n}, y\right)=D(v, y)$ for every $y \in X$.

\section{THE RESUlTS}

In this section, we shall prove dualistic partial metric version of Theorem 1.1. We state our main result as follows.

Theorem 3.1. Let $(X, D)$ be a complete dualistic partial metric space and $T: X \rightarrow X$ be a mapping satisfying

$$
\varphi(|D(T(x), T(y))|) \leq \varphi(M(x, y))-\psi(M(x, y)) \text { for all } x, y \in X
$$

where $M(x, y)=\max \left\{|D(x, y)|,\left|\frac{D(y, T(y))(1+D(x, T(x))}{1+D(x, y)}\right|\right\}$ and

$\varphi:[0, \infty) \rightarrow[0, \infty)$ is a continuous and monotone non-decreasing function with $\varphi(t)=0$ if and only if $t=0$ and $\psi:[0, \infty) \rightarrow[0, \infty)$ is a lower semi-continuous function with $\psi(t)=0$ if and only if $t=0$. Then $T$ has a unique fixed point.

Proof. Let $x_{0}$ be an initial point of $\mathrm{X}$ and let us define Picard iterative sequence $\left\{x_{n}\right\}$ by

$$
x_{n}=T\left(x_{n-1}\right) \text { for all } n \in \mathbb{N} \text {. }
$$

If there exists a positive integer $i$ such that $x_{i}=x_{i+1}$, then $x_{i}=x_{i+1}=T\left(x_{i}\right)$, so $x_{i}$ is a fixed point of $T$. In this case proof is complete. On the other hand if $x_{n} \neq x_{n+1}$ for all $n \in \mathbb{N}$, then from contractive condition (3.1) we have for $x_{n}, x_{n+1} \in X$

$$
\varphi\left(\left|D\left(T\left(x_{n-1}\right), T\left(x_{n}\right)\right)\right|\right) \leq \varphi\left(M\left(x_{n-1}, x_{n}\right)\right)-\psi\left(M\left(x_{n-1}, x_{n}\right)\right) .
$$

That is

$$
\varphi\left(\left|D\left(x_{n}, x_{n+1}\right)\right|\right) \leq \varphi\left(M\left(x_{n-1}, x_{n}\right)\right)-\psi\left(M\left(x_{n-1}, x_{n}\right)\right) .
$$


Where

$$
\begin{aligned}
M\left(x_{n-1}, x_{n}\right) & =\max \left\{\left|D\left(x_{n-1}, x_{n}\right)\right|,\left|\frac{D\left(x_{n}, x_{n+1}\right)\left(1+D\left(x_{n-1}, x_{n}\right)\right.}{1+D\left(x_{n-1}, x_{n}\right)}\right|\right\} . \\
& =\max \left\{\left|D\left(x_{n-1}, x_{n}\right)\right|,\left|D\left(x_{n}, x_{n+1}\right)\right|\right\} .
\end{aligned}
$$

If $\left|D\left(x_{n-1}, x_{n}\right)\right| \leq\left|D\left(x_{n}, x_{n+1}\right)\right|$, then $M\left(x_{n-1}, x_{n}\right)=\left|D\left(x_{n}, x_{n+1}\right)\right|$

and therefore (3.2) implies,

$$
\begin{aligned}
\varphi\left(\left|D\left(x_{n}, x_{n+1}\right)\right|\right) & \leq \varphi\left(\left|D\left(x_{n}, x_{n+1}\right)\right|\right)-\psi\left(\left|D\left(x_{n}, x_{n+1}\right)\right|\right) \\
& <\varphi\left(\left|D\left(x_{n}, x_{n+1}\right)\right|\right)
\end{aligned}
$$

Which is a contradiction due to the fact $\left|D\left(x_{n}, x_{n+1}\right)\right|>0$. Hence $M\left(x_{n-1}, x_{n}\right)=\left|D\left(x_{n-1}, x_{n}\right)\right|$. So in this case (3.2) gives,

$$
\varphi\left(\left|D\left(x_{n}, x_{n+1}\right)\right|\right)<\varphi\left(\left|D\left(x_{n-1}, x_{n}\right)\right|\right)
$$

which implies $\left|D\left(x_{n}, x_{n+1}\right)\right| \leq\left|D\left(x_{n-1}, x_{n}\right)\right|$.

Thus, $\left\{\left|D\left(x_{n}, x_{n+1}\right)\right|\right\}_{n \in \mathbb{N}}$ is a nonincreasing sequence of positive real numbers. There exists a number $L \geq 0$ such that $\lim _{n \rightarrow \infty}\left|D\left(x_{n}, x_{n+1}\right)\right|=L$. We claim that $L=0$. On contrary suppose that $L>0$ and taking upper limit of

$$
\varphi\left(\left|D\left(x_{n}, x_{n+1}\right)\right|\right) \leq \varphi\left(\left|D\left(x_{n-1}, x_{n}\right)\right|\right)-\psi\left(\left|D\left(x_{n-1}, x_{n}\right)\right|\right)
$$

we get

$$
\begin{aligned}
& \varphi(L) \leq \varphi(L)-\lim _{n \rightarrow \infty} \inf \psi\left(\left|D\left(x_{n-1}, x_{n}\right)\right|\right) . \\
& \varphi(L) \leq \varphi(L)-\psi(L)<\varphi(L) .
\end{aligned}
$$

Which is a contradiction, so $L=0$ and hence

$$
\lim _{n \rightarrow \infty}\left|D\left(x_{n}, x_{n+1}\right)\right|=0 \text { implies } \lim _{n \rightarrow \infty} D\left(x_{n}, x_{n+1}\right)=0
$$

Now to find $\lim _{n \rightarrow \infty}\left|D\left(x_{n}, x_{n}\right)\right|$, we use (3.1) again,

$$
\varphi\left(\left|D\left(T\left(x_{n-1}\right), T\left(x_{n-1}\right)\right)\right|\right) \leq \varphi\left(M\left(x_{n-1}, x_{n-1}\right)\right)-\psi\left(M\left(x_{n-1}, x_{n-1}\right)\right) .
$$


That is

$$
\varphi\left(\left|D\left(x_{n}, x_{n}\right)\right|\right) \leq \varphi\left(M\left(x_{n-1}, x_{n-1}\right)\right)-\psi\left(M\left(x_{n-1}, x_{n-1}\right)\right) .
$$

Where $\left.M\left(x_{n-1}, x_{n-1}\right)\right)=\max \left\{\left|D\left(x_{n-1}, x_{n-1}\right)\right|,\left|\frac{D\left(x_{n}, x_{n-1}\right)\left(1+D\left(x_{n-1}, x_{n}\right)\right.}{1+D\left(x_{n-1}, x_{n-1}\right)}\right|\right\}$.

If

$$
M\left(x_{n-1}, x_{n-1}\right)=\left|\frac{D\left(x_{n}, x_{n-1}\right)\left(1+D\left(x_{n-1}, x_{n}\right)\right.}{1+D\left(x_{n-1}, x_{n-1}\right)}\right|
$$

, then taking upper limit on (3.4) and using (3.3), we obtain,

$\lim _{n \rightarrow \infty} \varphi\left(\left|D\left(x_{n}, x_{n}\right)\right|\right) \leq 0$. This implies that $\lim _{n \rightarrow \infty} \varphi\left(\left|D\left(x_{n}, x_{n}\right)\right|\right)=0$. By continuity of $\varphi$, we have $\lim _{n \rightarrow \infty}\left|D\left(x_{n}, x_{n}\right)\right|=0$. Similarly if $M\left(x_{n-1}, x_{n-1}\right)=\left|D\left(x_{n-1}, x_{n-1}\right)\right|$, then

$$
\begin{gathered}
\varphi\left(\left|D\left(x_{n}, x_{n}\right)\right|\right) \leq \varphi\left(\left|D\left(x_{n-1}, x_{n-1}\right)\right|\right)-\psi\left(\left|D\left(x_{n-1}, x_{n-1}\right)\right|\right) . \\
\varphi\left(\left|D\left(x_{n}, x_{n}\right)\right|\right)<\varphi\left(\left|D\left(x_{n-1}, x_{n-1}\right)\right|\right) . \\
\text { it implies }\left|D\left(x_{n}, x_{n}\right)\right| \leq\left|D\left(x_{n-1}, x_{n-1}\right)\right| .
\end{gathered}
$$

Thus $\left\{\left|D\left(x_{n}, x_{n}\right)\right|\right\}_{n \in \mathbb{N}}$ is a nonincreasing sequence of positive real numbers and arguing like above, we get

$$
\lim _{n \rightarrow \infty} D\left(x_{n}, x_{n}\right)=0
$$

Since $d_{D}\left(x_{n}, x_{n+1}\right)=D\left(x_{n}, x_{n+1}\right)-D\left(x_{n}, x_{n}\right)$, so using (3.6), we get

$$
\lim _{n \rightarrow \infty} d_{D}\left(x_{n}, x_{n+1}\right)=0
$$

Now we show that $\left\{x_{n}\right\}$ is a Cauchy sequence in $\left(X, d_{D}^{S}\right)$. For this we have to show that $\lim _{n, m \rightarrow \infty} d_{D}^{s}\left(x_{n}, x_{m}\right)=0$. That is $\lim _{n, m \rightarrow \infty} d_{D}\left(x_{n}, x_{m}\right)=0=\lim _{n, m \rightarrow \infty} d_{D}\left(x_{m}, x_{n}\right)$. Suppose on contrary that $\lim _{n, m \rightarrow \infty} d_{D}\left(x_{n}, x_{m}\right) \neq 0$. Then there exists $\varepsilon>0$ for which we can find two sub sequences $\left\{x_{n_{k}}\right\},\left\{x_{m_{k}}\right\}$ of $\left\{x_{n}\right\}$ such that $n_{k}$ is smallest index for which

$$
n_{k}>m_{k} \text { and } d_{D}\left(x_{n_{k}}, x_{m_{k}}\right) \geq \varepsilon
$$

This means that

$$
d_{D}\left(x_{n_{k}-1}, x_{m_{k}}\right)<\varepsilon
$$


Now using (3.8) and (3.9), we have

$$
\begin{aligned}
\varepsilon \leq d_{D}\left(x_{n_{k}}, x_{m_{k}}\right) & \leq d_{D}\left(x_{n_{k}}, x_{n_{k}-1}\right)+d_{D}\left(x_{n_{k}-1}, x_{m_{k}}\right) \\
& \leq d_{D}\left(x_{n_{k}}, x_{n_{k}-1}\right)+\varepsilon
\end{aligned}
$$

Taking $\lim _{k \rightarrow \infty}$ and using (3.7), we obtain

$$
\lim _{k \rightarrow \infty} d_{D}\left(x_{n_{k}}, x_{m_{k}}\right)=\varepsilon
$$

Due to triangular inequality, we get

$$
\begin{aligned}
d_{D}\left(x_{n_{k}}, x_{m_{k}}\right) & \leq d_{D}\left(x_{n_{k}}, x_{n_{k}-1}\right)+d_{D}\left(x_{n_{k}-1}, x_{m_{k}}\right) . \\
& \leq d_{D}\left(x_{n_{k}}, x_{n_{k}-1}\right)+d_{D}\left(x_{n_{k}-1}, x_{m_{k}-1}\right)+d_{D}\left(x_{m_{k}-1}, x_{m_{k}}\right) .
\end{aligned}
$$

But then

$$
\begin{aligned}
d_{D}\left(x_{n_{k}-1}, x_{m_{k}-1}\right) & \leq d_{D}\left(x_{n_{k}-1}, x_{n_{k}}\right)+d_{D}\left(x_{n_{k}}, x_{m_{k}-1}\right) . \\
& \leq d_{D}\left(x_{n_{k}-1}, x_{n_{k}}\right)+d_{D}\left(x_{n_{k}}, x_{m_{k}}\right)+d_{D}\left(x_{m_{k}}, x_{m_{k}-1}\right)
\end{aligned}
$$

Taking $\lim _{k \rightarrow \infty}$ in above expressions and using (3.7), (3.10) we obtain,

$$
\lim _{k \rightarrow \infty} d_{D}\left(x_{n_{k}-1}, x_{m_{k}-1}\right)=\varepsilon
$$

Following (3.1) for $x_{n_{k}} \neq x_{m_{k}}$, we have

$$
\varphi\left(\left|D\left(T\left(x_{n_{k}-1}\right), T\left(x_{m_{k}-1}\right)\right)\right|\right) \leq \varphi\left(M\left(x_{n_{k}-1}, x_{m_{k}-1}\right)\right)-\psi\left(M\left(x_{n_{k}-1}, x_{m_{k}-1}\right)\right) .
$$

That is

$$
\varphi\left(\left|D\left(x_{n_{k}}, x_{m_{k}}\right)\right|\right) \leq \varphi\left(M\left(x_{n_{k}-1}, x_{m_{k}-1}\right)\right)-\psi\left(M\left(x_{n_{k}-1}, x_{m_{k}-1}\right)\right) .
$$

Where

$$
M\left(x_{n_{k}-1}, x_{m_{k}-1}\right)=\max \left\{\left|D\left(x_{n_{k}-1}, x_{m_{k}-1}\right)\right|,\left|\frac{D\left(x_{m_{k}-1}, x_{m_{k}}\right)\left(1+D\left(x_{n_{k}-1}, x_{n_{k}}\right)\right.}{1+D\left(x_{n_{k}-1}, x_{n_{k}-1}\right)}\right|\right\}
$$

By using (3.10) and (3.11), we deduce that

$$
\lim _{k \rightarrow \infty} M\left(x_{n_{k}-1}, x_{m_{k}-1}\right)=\varepsilon .
$$


Now applying upper limit on (3.12) and using (3.10), (3.11) along with properties of $\varphi, \psi$ we get

$$
\varphi(\varepsilon) \leq \varphi(\varepsilon)-\lim _{k \rightarrow \infty} \inf \psi\left(M\left(x_{n_{k}-1}, x_{m_{k}-1}\right)\right) .
$$

That is $\varphi(\varepsilon)<\varphi(\varepsilon)$, a contradiction and therefore $\lim _{n, m \rightarrow \infty} d_{D}\left(x_{n}, x_{m}\right)=0$. Similarly we can prove that $\lim _{n, m \rightarrow \infty} d_{D}\left(x_{m}, x_{n}\right)=0$.

Hence $\lim _{n, m \rightarrow \infty} d_{D}^{s}\left(x_{n}, x_{m}\right)=0$ which entails that $\left\{x_{n}\right\}$ is a Cauchy sequence in $\left(X, d_{D}^{s}\right)$. Since $(X, D)$ is a complete dualistic partial metric space, so by Lemma $2.5\left(X, d_{D}^{S}\right)$ is also a complete metric space. Thus, there exists $v$ in $X$ such that $\lim _{n \rightarrow \infty} d_{D}^{s}\left(x_{n}, v\right)=0$, again from Lemma 2.5, we get

$$
\lim _{n \rightarrow \infty} d_{D}^{S}\left(x_{n}, v\right)=0 \Longleftrightarrow \lim _{n \rightarrow \infty} D\left(v, x_{n}\right)=D(v, v)=\lim _{n, m \rightarrow \infty} D\left(x_{n}, x_{m}\right) .
$$

Since $\lim _{n, m \rightarrow \infty} d_{D}\left(x_{n}, x_{m}\right)=0$, thus, $\lim _{n, m \rightarrow \infty} D\left(x_{n}, x_{m}\right)=0$. From (3.14) $D(v, v)=0=$ $\lim _{n \rightarrow \infty} D\left(v, x_{n}\right)$. Now we prove that $v$ is a fixed point of $T$. On contrary suppose that $v \neq T(v)$, then using (3.1) and Lemma 2.5 we have

$$
\varphi\left(\mid D\left(x_{n}, T(v) \mid\right)=\varphi\left(\left|D\left(T\left(x_{n-1}\right), T(v)\right)\right|\right) \leq \varphi\left(M\left(x_{n-1}, v\right)\right)-\psi\left(M\left(x_{n-1}, v\right)\right) .\right.
$$

That is

$$
\varphi\left(\left|D\left(x_{n}, T(v)\right)\right|\right) \leq \varphi\left(M\left(x_{n-1}, v\right)\right)-\psi\left(M\left(x_{n-1}, v\right)\right)
$$

Letting $n \rightarrow \infty$ and using properties of $\varphi, \psi$ we get $\varphi(D(v, T(v)))<\varphi(D(v, T(v)))$. Which is a contradiction as $D(v, T(v)) \geq 0$. Hence $v=T(v)$ that is $v$ is a fixed point of $T$. Finally, we shall prove the uniqueness. Suppose that $\omega$ is another xed point of $T$ such that $v \neq \omega$, then from (3.1),we have

$$
\varphi(|D(v, \omega)|) \leq \varphi(M(v, \omega))-\psi(M(v, \omega)) .
$$

which implies that

$$
\varphi(|D(v, \omega)|)<\varphi(|D(v, \omega)|)
$$

A contradiction, hence $v=\omega$. So $T$ has a unique fixed point in $X$.

In Theorem 3.1, if we take $\varphi(t)=t$ and $\psi(t)=(1-h) t$ where $h \in[0,1[$ and $t \geq 0$. Then we have following result 
Corollary 3.2. Let $(X, D)$ be a complete dualistic partial metric space and $T: X \rightarrow X$ be $a$ mapping satisfying

$$
|D(T(x), T(y))| \leq h \max \left\{|D(x, y)|,\left|\frac{D(y, T(y))(1+D(x, T(x))}{1+D(x, y)}\right|\right\} \text { for all } x, y \in X,
$$

Then $T$ has a unique fixed point.

For if $D(x, y) \in \mathbb{R}_{0}^{+}$for all $x, y \in X$, then $D(x, y)=p(x, y)$. The partial metric version of Corollary 3.2 can be obtained as follows:

Corollary 3.3. Let $(X, p)$ be a complete partial metric space and $T: X \rightarrow X$ be a mapping satisfying

$$
p(T(x), T(y)) \leq h \max \left\{p(x, y), \frac{p(y, T(y))(1+p(x, T(x))}{1+p(x, y)}\right\} \text { for all } x, y \in X,
$$

Then T has a unique fixed point.

Now if

$$
\max \left\{|D(x, y)|,\left|\frac{D(y, T(y))(1+D(x, T(x))}{1+D(x, y)}\right|\right\}=|D(x, y)|,
$$

then the result obtained by Valero [7] can be viewed as a special case of Corollary 3.2.

Corollary 3.4. [7] Let $(X, D)$ be a complete dualistic partial metric space and let $T: X \rightarrow X$ be a self-mapping such that there exists $\alpha \in[0,1[$ satisfying

$$
|D(T(x), T(y))| \leq \alpha|D(x, y)|
$$

for all $x, y \in X$. Then Thas a unique fixed point $x^{*} \in X$. Moreover, $D\left(x^{*}, x^{*}\right)=0$ and the Picard iterative sequence $\left\{T^{n}\left(x_{0}\right)\right\}_{n \in \mathbb{N}}$ converges to $x^{*}$ with respect to $\tau\left(d_{D}^{s}\right)$, for every $x \in X$.

Remark 3.5. As every dualistic partial metric $D$ is an extension of partial metric $p$, therefore, Theorem 3.1 is an extension of Theorem 1.1. Consequently, Corollary 3.2 generalizes Corollary 3.3 .

There arises the following natural question: 
Whether the contractive condition in the statement of Corollary 3.2 can be replaced by the contractive condition in Corollary 3.3. Following example will give the negative answer to this question.

Example 3.6. Consider the complete dualistic partial metric $\left(\mathbb{R}, D_{\vee}\right)$. Define the self-mapping $T_{0}: \mathbb{R} \rightarrow \mathbb{R}$ by

$$
T_{0}(x)=\left\{\begin{array}{rr}
0 & \text { if } x \neq 0 \\
-1 & \text { if } x=0
\end{array} .\right.
$$

It is easy to check that fixed point free mapping $T_{0}$ does not satisfy the contractive condition in the statement of Corollary 3.2. Indeed,

$$
\left.1=\left|D_{\vee}(-1,-1)\right|=\left|D_{\vee}\left(T_{0}(0), T_{0}(0)\right)\right|\right)>h M(0,0) .
$$

Where

$$
M(0,0)=\max \left\{\left|D_{\vee}(0,0)\right|,\left|\frac{D_{\vee}\left(0, T_{0}(0)\right)\left(1+D_{\vee}\left(0, T_{0}(0)\right)\right)}{1+D_{\vee}(0,0)}\right|\right\} .
$$

Nevertheless, the contractive condition in the statement of Corollary 3.3 holds true. Indeed,

$$
\left.-1=p_{\vee}(-1,-1)=p_{\vee}\left(T_{0}(0), T_{0}(0)\right) \mid\right) \leq h M(0,0) .
$$

Where

$$
M(0,0)=\max \left\{p_{\vee}(0,0), \frac{p_{\vee}\left(0, T_{0}(0)\right)\left(1+p_{\vee}\left(0, T_{0}(0)\right)\right)}{1+p_{\vee}(0,0)}\right\}=0
$$

Example 3.7. Let $\left(\mathbb{R}, D_{\vee}\right)$ be a complete dualistic partial metric space. Define the self-mapping $T_{1}: \mathbb{R} \rightarrow \mathbb{R}$ by

$$
T_{1}(x)=\left\{\begin{array}{rr}
0 & \text { if } x=0 \\
-1 & \text { if } x \geq 2
\end{array} .\right.
$$

The mapping $T_{1}$ has a unique fixed point $x=0$. It is easy to check that $T_{1}$ satisfies the contractive condition in the statement of Corollary 3.2. Indeed, for all $x \geq y \geq 2$ and $h>\frac{1}{2}$

$$
\begin{aligned}
& \left|D_{\vee}\left(T_{1}(x), T_{1}(y)\right)\right| \leq h \max \left\{\left|D_{\vee}(x, y)\right|,\left|\frac{D_{\vee}\left(y, T_{1}(y)\right)\left(1+D_{\vee}\left(x, T_{1}(x)\right)\right.}{1+D_{\vee}(x, y)}\right|\right\} \\
& 1=\left|D_{\vee}(-1,-1)\right| \leq h x
\end{aligned}
$$


holds. Also note that for $x=0=y$ the contractive condition in the statement of Corollary 3.2 trivially holds.

\section{APPLICATION TO FUNCTIONAL EQUATIONS}

As an application of our fixed point result [Corollary 3.2], we present the study about the existence and uniqueness of the solution of functional equations. we introduce some notations for the sake of convenience.

$$
\begin{aligned}
S & =\text { State space. } \\
W & =\text { Decision space. } \\
B(S) & =\text { Space of bounded functions. } \\
c_{n} & =\text { Sequence of real numbers such that } \lim _{n \rightarrow \infty}\left|c_{n}\right|=0 . \\
g & : S \times W \rightarrow \mathbb{R} . \\
F_{n} & : S \times W \times \mathbb{R} \rightarrow \mathbb{R} \text { where } n=0,1,2,3, \cdots . \\
\phi & : S \times W \rightarrow S .
\end{aligned}
$$

In the following we shall prove the existence and uniqueness of solution of functional equation appearing in dynamic programming. (for example see [1])

$$
u(x)=\sup _{y \in W}\left\{g(x, y)+F_{n}(x, y, u(\phi(x, y)))\right\} \forall x \in S
$$

We observe that the spaces $\left(B(S),\|\cdot\|_{\infty}\right)$ is a Banach space and distance function in $B(S)$ is defined by

$$
d_{\infty}(u, v)=\sup _{x \in S}|u(x)-v(x)| \forall u, v \in B(S)
$$

where as for dualistic partial metric space distant function is given by

$$
D_{\infty}(u, v)=d_{\infty}(u, v)+c_{n}, \forall u, v \in B(S)
$$

In calculations following two lemmas will be helpful. 
Lemma 4.1. [1] Let $G, H: S \rightarrow \mathbb{R}$ be two bounded functions then,

$$
\left|\sup _{x \in S} G(x)-\sup _{x \in S} H(x)\right| \leq \sup _{x \in S}|G(x)-H(x)| .
$$

Lemma 4.2. [1] Let

(1) $\mathrm{g}, \mathrm{F}$ be bounded functions.

(2) $\exists k>0$ such that $\forall t, r \in \mathbb{R}, x \in S$ and $y \in W$.

$$
\left|F_{n}(x, y, t)-F_{n}(x, y, r)\right| \leq k|t-r| .
$$

Then the operator $R: B(S) \rightarrow B(S)$ defined by

$$
(R u)(x)=\sup _{y \in W}\left\{g(x, y)+F_{n}(x, y, u(\phi(x, y)))\right\}
$$

is well define.

Now we present our next result.

Theorem 4.3. Let all the conditions of lemma 4.2 be satisfied and for $n \rightarrow \infty$

$$
\left|F_{n}(x, y, u)-F_{n}(x, y, v)\right| \leq h \max \left\{\left|D_{\infty}(u, v)\right|,\left|\frac{D_{\infty}(v, R v)\left(1+D_{\infty}(u, R u)\right.}{1+D_{\infty}(u, v)}\right|\right\}
$$

Then the functional equation (4.1) has unique solution.

Proof. Let $R: B(S) \rightarrow B(S)$ be an operator as defined in lemma 4.2. We shall show that $\mathrm{R}$ satisfies contractive condition (3.15). Indeed by lemma 4.1, for all $u, v \in B(S)$.

$$
\begin{aligned}
|(R u)(x)-(R v)(x)| & =\left|\sup _{y \in W}\left\{g(x, y)+F_{n}(x, y, u(\phi(x, y)))\right\}-\sup _{y \in W}\left\{g(x, y)+F_{n}(x, y, v(\phi(x, y)))\right\}\right| \\
& \leq \sup _{y \in W}\left|g(x, y)+F_{n}(x, y, u(\phi(x, y)))-g(x, y)-F_{n}(x, y, v(\phi(x, y)))\right| \\
& \leq \sup _{y \in W}\left|F_{n}(x, y, u(\phi(x, y)))-F_{n}(x, y, v(\phi(x, y)))\right| .
\end{aligned}
$$

Therefore,

$$
\begin{aligned}
\left|D_{\infty}(R u, R v)\right| & =\left|\sup _{x \in S}\right|(R u)(x)-(R v)(x)\left|+c_{n}\right| \\
& \leq \sup _{x \in S}|(R u)(x)-(R v)(x)|+\left|c_{n}\right| \\
& \leq \sup _{y \in W}\left|F_{n}(x, y, u(\phi(x, y)))-F_{n}(x, y, v(\phi(x, y)))\right|+\left|c_{n}\right|
\end{aligned}
$$


When $n \rightarrow \infty$ then by (4.2) we obtain,

$$
\left|D_{\infty}(R u, R v)\right| \leq h \max \left\{\left|D_{\infty}(u, v)\right|,\left|\frac{D_{\infty}(v, R v)\left(1+D_{\infty}(u, R u)\right.}{1+D_{\infty}(u, v)}\right|\right\}+\lim _{n \rightarrow \infty}\left|c_{n}\right| .
$$

Finally, definition of $c_{n}$ gives

$$
\left|D_{\infty}(R u, R v)\right| \leq h \max \left\{\left|D_{\infty}(u, v)\right|,\left|\frac{D_{\infty}(v, R v)\left(1+D_{\infty}(u, R u)\right.}{1+D_{\infty}(u, v)}\right|\right\} .
$$

Hence, R satisfies all the conditions of Corollary 3.2. Thus there exists a unique solution of (4.1) $u_{0} \in B(S)$ such that $R u_{0}=u_{0}$.

Remark 4.4. Significance of the above results lies in the fact that these results are true for all real numbers whereas such results proved in partial metric spaces are only true for positive real numbers.

\section{REFERENCES}

[1] R. Bellman, E.S. Lee: Functional equations in dynamic programming. Aeq. Math., 17 (1978), 1-18.

[2] N.V. Can, N.X. Thuan: Fixed point theorem for generalized weak contractions involving rational expressions. Open Journal of Mathematical Modeling, 1 (2013), 29-33.

[3] B.K. Dass, S. Gupta: An extension of Banach contraction principle through rational expressions. Indian J. Pure Appl. Math., 6 (1975), 1455-1458.

[4] J. Harjani, B. Lopez, K. Sadarangani, A fixed point theorem for mappings satisfying a contractive condition of rational type on a partially ordered metric space, Abstr. Appl. Anal. (2010), Art. ID 190701.

[5] E.karapinar, W.Shatanawi, K.Tas: Fixed point theorem on partial metric spaces involving rational expressions. Miskolc Mathematical Notes, HU e-ISSN 1787-2413,Vol. 14 (2013), No. 1, pp. 135-142.

[6] S.G. Matthews, Partial Metric Topology, in proceedings of the $11^{t} h$ Summer Conference on General Topology and Applications, 728 (1995), 183-197, The New York Academy of Sciences.

[7] S.Oltra and O.Valero, Banach's fixed point theorem for partial metric spaces, Rend. Ist. Mat. Univ. Trieste 36(2004),17-26.

[8] S.J.O'Neill, Partial Metric, Valuations and Domain Theory. Annals of the New York Academy of Science, 806 (1996), 304-315.

[9] O. Valero, On Banach fixed point theorems for partial metric spaces, Applied General Topology, 6(2)(2005). 